Article

\title{
A Ginzburg-Landau Type Energy with Weight and with Convex Potential Near Zero
}

\author{
Rejeb Hadiji ${ }^{1}$ and Carmen Perugia ${ }^{2, *}$
}

1 Laboratoire d'Analyse et de Mathématiques Appliquées, LAMA, Université Paris-Est, UMR 8050, UPEC, F-94010 Créteil, France; rejeb.hadiji@u-pec.fr

2 Dipartimento di Scienze e Tecnologie, Universitá del Sannio, Via de Sanctis, 82100 Benevento, Italy

* Correspondence: cperugia@unisannio.it

Received: 21 May 2020; Accepted: 16 June 2020; Published: 18 June 2020

check for updates

\begin{abstract}
In this paper, we study the asymptotic behavior of minimizing solutions of a Ginzburg-Landau type functional with a positive weight and with convex potential near 0 and we estimate the energy in this case. We also generalize a lower bound for the energy of unit vector field given initially by Brezis-Merle-Rivière.
\end{abstract}

Keywords: Ginzburg-Landau functional; lower bound; variational problem

MSC: 35Q56; 35J50; 35B25

\section{Introduction}

Let $G$ be a bounded, simply connected and smooth domain of $\mathbf{R}^{2}, g: \partial G \rightarrow S^{1}$ a smooth boundary data of degree $d$ and $p$ a smooth positive function on $\bar{G}$. We set

$$
p_{0}=\min \{p(x): x \in \bar{G}\}
$$

and $\Lambda=p^{-1}\left(p_{0}\right)$. Let us consider a $C^{2}$ functional $J: \mathbf{R} \rightarrow[0, \infty)$ satisfying the following conditions :

Hypothesis $1(\mathrm{H} 1) . J(0)=0$ and $J(t)>0$ on $(0, \infty)$.

Hypothesis $2(\mathrm{H} 2) . J^{\prime}(t)>0$ on $(0,1]$.

Hypothesis $3(\mathrm{H} 3)$. there exists $\rho_{0}>0$ such that $J^{\prime \prime}(t)>0$ on $\left(0, \rho_{0}\right)$.

For each $\varepsilon>0$ let $u_{\varepsilon}$ be a minimizer for the following Ginzburg-Landau type functional

$$
E_{\varepsilon}(u)=\int_{G} p|\nabla u|^{2} d x+\frac{1}{\varepsilon^{2}} \int_{G} J\left(1-|u|^{2}\right) d x
$$

defined on the set

$$
H_{g}^{1}(G, \mathbf{C})=\left\{u \in H^{1}(G, \mathbf{C}): u=g \text { on } \partial G\right\} .
$$

It is easy to prove that $\min _{u \in H_{g}^{1}(G, C)} E_{\varepsilon}(u)$ is achieved by some smooth $u_{\varepsilon}$ which satisfies

$$
\left\{\begin{array}{cc}
-\operatorname{div}\left(p \nabla u_{\varepsilon}\right)=\frac{1}{\varepsilon^{2}} j\left(1-\left|u_{\varepsilon}\right|^{2}\right) u_{\varepsilon} & \text { in } G \\
u_{\varepsilon}=g & \text { on } \partial G,
\end{array}\right.
$$


where $j(t)=J^{\prime}(t)$. In this paper, we are interested in studying the asymptotic behavior of $u_{\varepsilon}$ and estimate the energy $E_{\varepsilon}\left(u_{\varepsilon}\right)$ as $\varepsilon \rightarrow 0$ under the assumptions that $p$ has a finite number of local minima $b_{1}, \ldots, b_{N}$ all lying in $G$ and that it behaves in a "good" way in a neighborhood of each of its minima. More precisely, throughout this paper we shall assume

$$
\Lambda=\left\{b_{1}, \ldots, b_{N}\right\} \subset G
$$

and there exist real numbers $\alpha_{k}, \beta_{k}, s_{k}$ satisfying $0<\alpha_{k} \leq \beta_{k}$ and $s_{k}>1$ such that

$$
\alpha_{k}\left|x-b_{k}\right|^{s_{k}} \leq p(x)-p_{0} \leq \beta_{k}\left|x-b_{k}\right|^{s_{k}}
$$

in a neighborhood of $b_{k}$ for every $1 \leq k \leq N$.

The presence of a non-constant weight function is motivated by the problem of pinning the vortices of $u_{\varepsilon}$ to some restricted sites, see $[11,13,20]$ for more detailed physical motivations. Indeed, in general, the study of the minimization of the energy functional Problem (2) and its particular form is motivated by pinning phenomena in superconductivity that attract vortices to some sites. In [1], the authors show that in presence of an applied magnetic field, if the applied fields reach a critical value, these sites are attracted away from the interior, the pinning effect breaks down and vortices appear in the interior. In [22], the authors consider a model of a superconductor subjected to an applied electric current and electromagnetic field and containing impurities. They study a mixed heat and Schrödinger Ginzburg-Landau evolution equation on a bounded two-dimensional domain with an electric current applied on the boundary and a pinning potential term. Other models are considered in [2], where the authors treat the structure of symmetric vortices in a Ginzburg-Landau model of high-temperature superconductivity and antiferromagnetism. In [4], the authors give an analysis of minimizers of the Lawrence-Doniach energy for superconductors in applied fields.

Please note that in [19], the author investigates a different type of generalization for the standard Ginzburg-Landau problem, taking the weight $p=1$ and allowing the potential to vanish on a larger set.

Our way of act provides an approach to various proofs related to stationary GinzburgLandau vortices.

In this paper, without loss of generality, we assume $d \geq 0$. By the way we treat only the case $d>0$, being the case $d=0$ trivial.

The case when $J(|u|)=\frac{\left(1-|u|^{2}\right)^{2}}{4}$ and $p=\frac{1}{2}$ corresponding to the Ginzburg-Landau energy, was studied by several authors since the groundbreaking works of Béthuel-Brezis and Hélein. More precisely they dealt with the case with boundary data satisfying $d=0$ and $d \neq 0$ respectively in $[5,6]$. In this latter work, the case of $G$ star shaped was treated. Eventually in [23], Struwe gave an argument which works for an arbitrary domain and later del Pino and Felmer in [12] gave a very simple argument for reducing the general case to the star shaped one. More in particular the method of Struwe is found to be very useful for the case of non-constant $p$. We note that in [14] we study the effect of the presence of $|u|$ in the weight $p(x, u)=p_{0}+s|x|^{k}|u|^{l}$ where $s$ is small, $k \geq 0$ and $l \geq 0$.

The case when $J(|u|)=\frac{\left(1-|u|^{2}\right)^{2}}{4}$ and $p$ blue is not a constant function was studied in [3,7-9]. More precisely in [7-9] the authors considered the cases card $\Lambda=1$ and $d \geq 1$, card $\Lambda \geq d$ and the case where $p$ has minima on the boundary of the domain. In the first case they highlight a singularity of degree greater than 1 when $d>1$. More precisely, if $\Lambda=\{b\} \subset G$, they proved

$$
u_{\varepsilon_{n}} \rightarrow u_{*}=e^{i \phi}\left(\frac{z-b}{|z-b|}\right)^{2 d} \quad \text { in } \quad C_{l o c}^{1, \alpha}(\bar{G} \backslash\{b\}),
$$

where $\phi$ is determined by the boundary data $g$.

In the second case, they showed that actually $N=d$, the degree around each $b_{k}$ is equal to 1 and for a subsequence $\varepsilon_{n} \rightarrow 0$ 


$$
u_{\varepsilon_{n}} \rightarrow u_{*}=e^{i \phi} \prod_{j=1}^{d} \frac{z-b_{j}}{\left|z-b_{j}\right|} \quad \text { in } \quad C_{l o c}^{1, \alpha}\left(\bar{G} \backslash\left\{b_{1}, \ldots, b_{d}\right\}\right)
$$

the configuration $\left\{b_{1}, \ldots, b_{d}\right\}$ being minimizing for a certain renormalized energy defined in $\Lambda^{d}$. Moreover, they proved the asymptotic behavior $E_{\varepsilon}\left(u_{\varepsilon}\right)=\pi p_{0} d|\log \varepsilon|+O(1)$. In the third case, the authors considered the situation when the weight has both minima in the domain and on the boundary. In [3], the authors studied the case card $\Lambda<d$ and established the convergence of a subsequence $u_{\varepsilon_{n}} \rightarrow u_{*}$ in $C_{l o c}^{1, \alpha}\left(\bar{G} \backslash\left\{b_{1}, \ldots, b_{N}\right\}\right)$ for every $\alpha<1$, where the $N$ distinct points $\left\{b_{1}, \ldots, b_{N}\right\}$ lie in $\Lambda$ and $u_{*} \in C^{\infty}\left(\bar{G} \backslash\left\{b_{1}, \ldots, b_{N}\right\}, S^{1}\right)$ is a solution of

$$
-\operatorname{div}\left(p \nabla u_{*}\right)=p\left|\nabla u_{*}\right|^{2} u_{*} \text { in } \bar{G} \backslash\left\{b_{1}, \ldots, b_{N}\right\}, u_{*}=g \text { on } \partial G
$$

Moreover, the degree $d_{k}$ of $u_{*}$ around each $b_{k}$ satisfies $d_{k} \geq 1$ and $\sum_{k=1}^{N} d_{k}=d$.

In the current paper we will suppose that card $\Lambda=N<d$ as this is the more interesting case. Indeed, as already observed in [3], singularities of degree $>1$ must occur and in some cases they could be on the boundary. Following the same argument as in [5] or in [3], we prove that $u_{\varepsilon_{n}}$ has its zeros located in $d$ discs, called "bad discs", with radius $\lambda \varepsilon_{n}$ where $\lambda>0$. Outside this discs $\left|u_{\varepsilon_{n}}\right|$ is close to 1 . For $n$ large each bad disc contains exactly one zero. Thus, there are exactly $d_{k}$ zeros approaching each $b_{k}$ $($ as $n \rightarrow \infty)$. In the case $d_{k}>1$ (this must be the case of at least one $k$ if $N<d$ ), one expects to observe an "interaction energy" between zeros approaching the same limit $b_{k}$. A complete understanding of this process requires a study of the mutual distances between zeros of $u_{\varepsilon_{n}}$ which approach the same $b_{k}$. It turns out that these distances depend in a crucial way on the behavior of the weight function $p$ around its minima points. In [3], where $s_{k}=2$ and $J(|u|)=\frac{\left(1-|u|^{2}\right)^{2}}{4}$, it is showed that each $b_{k}$ with $d_{k}>1$ contributes an additional term to the energy, namely $\pi p_{0}\left(d_{k}^{2}-d_{k}\right) \log \left(|\log \varepsilon|^{\frac{1}{2}}\right)$ which is precisely the mentioned interaction energy. The method of $[5,6,23]$ can be adapted without any difficulties to the case of $J$ satisfying $(H 1) \div(H 3)$ with a zero of finite order at $t=0$. This applies for example to $J(t)=|t|^{l}, \forall l \geq 2$.

In our paper, due to the presence of a non-constant weight and a potential with zero of infinite order at $t=0$, the energy cost of each vortex of degree $d_{k}>1$ is much less than the previous one. Indeed, a precise computation of the energy around a minimum of the weight $p$, in the spirit of [3,7-9] will imply that certain potentials with sufficiently slow growth allow for a vortex energy that is not $2 \pi d_{k}|\log \varepsilon|+O(1)$ but instead

$$
2 \pi p_{0} d_{k}|\log \varepsilon|+2 \pi p_{0} \frac{d_{k}^{2}-d_{k}}{s_{k}} \log |\log \varepsilon|-2 \pi p_{0} d_{k} I\left(\frac{1}{\varepsilon}(|\log \varepsilon|)^{-\frac{1}{s_{k}}}\right)+o\left(I\left((|\log \varepsilon|)^{\frac{1}{s_{k}}}\right)\right),
$$

where the quantity

$$
I(R)=\frac{1}{2} \int_{\frac{1}{R^{2}}}^{j\left(\rho_{0}\right)} \frac{j^{-1}(t)}{t} d t
$$

will play an important role (see Section 3).

For the sake of clarity, let us give some natural example of the situation which we are studying which is only one very particular case among our general assumptions:

$$
J(t)=J_{h}(t)= \begin{cases}\exp \left(-1 / t^{h}\right) & \text { for } t>0 \\ 0 & \text { for } t \leq 0\end{cases}
$$

for $h>0$. Clearly, $J$ satisfies $(H 1)-(H 2)-(H 3)$. So, for example, for $J_{1}$ we find $I(R)=\frac{1}{2} \log \log R+$ $O(1)$, (see the Appendix Proposition 1.4 in [15]), and the vortex energy in this case reads: 


$$
2 \pi p_{0} d_{k}\left(|\log \varepsilon|-\frac{1}{2} \log |\log \varepsilon|\right)+2 \pi p_{0} \frac{d_{k}^{2}-d_{k}}{s_{k}} \log |\log \varepsilon|+O(1)
$$

Let us finally point out that it could also be interesting for our problem to give a precise asymptotic behavior of the term $\left.o\left(I(|\log \varepsilon|)^{\frac{1}{s_{k}}}\right)\right)$ in (7). At the moment, this question is not yet fully understood, since it is related to renormalized energy introduced in [8] (see also [3]).

Another interesting question is to study our problem (2) with the presence of an applied magnetic field. We guess it would be object of a forecoming papers.

The paper is organized as follows. In Section 2, we state our main result. In Section 3 we recall some definitions and results contained in [15]. Section 4 is devoted to prove the generalization of Theorem 4 of [10] which will be useful for obtaining a precise lower bound of the energy for our case. In Section 5 we prove our main result, namely Theorem 1, by stating an upper and a lower bound for the energy (2). Finally, as a corollary of upper and lower bounds of the energy, we find an estimate of the mutual distances between bad discs approaching the same singularity $b_{k}$.

\section{Statement of the Main Result}

Our main theorem describes the asymptotic behavior of the minimizers of the Ginzburg-Landau type functional (2) and their energies.

Theorem 1. For each $\varepsilon>0$, let $u_{\varepsilon}$ be a minimizer for the energy (2) over $H_{g}^{1}(G, \mathbf{C})$, with $G, g$ as above, $d>0$ and $J$ satisfying $(\mathrm{H} 1) \div(\mathrm{H} 3)$.

(i) For a subsequence $\varepsilon_{n} \rightarrow 0$ we have

$$
u_{\varepsilon_{n}} \rightarrow u_{*}=e^{i \phi} \prod_{j=1}^{N}\left(\frac{z-b_{j}}{\left|z-b_{j}\right|}\right)^{d_{j}} \text { in } C_{l o c}^{1, \alpha}\left(\bar{G} \backslash\left\{b_{1}, \ldots, b_{N}\right\}\right)
$$

for every $\alpha<1$, where the $N$ distinct points $\left\{b_{1}, \ldots, b_{N}\right\}$ lie in $\Lambda, \Sigma_{j=1}^{N} d_{j}=d$ and $\phi$ is a smooth harmonic function determined by the requirement $u_{*}=g$ on $\partial G$.

(ii) Setting

$$
I(R)=\frac{1}{2} \int_{\frac{1}{R^{2}}}^{j\left(\rho_{0}\right)} \frac{j^{-1}(t)}{t} d t
$$

we have

$$
\begin{aligned}
E_{\varepsilon_{n}}\left(u_{\varepsilon_{n}}\right)= & 2 \pi p_{0} d \log \frac{1}{\varepsilon_{n}}+2 \pi p_{0}\left(\Sigma_{k=1}^{N} \frac{d_{k}^{2}-d_{k}}{s_{k}}\right) \log \log \frac{1}{\varepsilon_{n}} \\
& -2 \pi p_{0} d I\left(\frac{1}{\varepsilon_{n}}\left(\log \frac{1}{\varepsilon_{n}}\right)^{-\frac{1}{s_{k}}}\right)+o\left(I\left(\left(\log \frac{1}{\varepsilon_{n}}\right)^{\frac{1}{s_{k}}}\right)\right) .
\end{aligned}
$$

As it is showed in [15], $\lim _{R \rightarrow \infty} \frac{I(R)}{\log R}=0$ hence the leading term in the energy is always of order $o(|\log \varepsilon|)$. Moreover, it is easy to see that $I(R)$ is a positive, monotone increasing, concave function of $\log R$ for $R$ large (see [15]). The proof of Theorem 1 consists of two main ingredients: the method of Struwe [23], as used also in [3] in order to locate the "bad discs", (i.e., a finite collection of discs of radius $O(\varepsilon)$ which cover the set $\left.\left\{x:\left|u_{\varepsilon}(x)<\frac{1}{2}\right|\right\}\right)$ and the generalization of a result of Brezis, Merle and Rivière [10] which will play an important role in finding the lower bound of the energy. More precisely in Theorem 2, we will bound from below the energy of a regular map defined away from some points $a_{1}, a_{2}, \ldots, a_{m}$ in $B_{R}(0)$ such that $0<a \leq|u| \leq 1$ in $\Omega, \operatorname{deg}\left(u, \partial B_{R}\left(a_{j}\right)=d_{j}\right.$ and with a bound potential by using the reference map $u_{0}(z)=\left(\frac{z-a_{1}}{\left|z-a_{1}\right|}\right)^{d_{1}}\left(\frac{z-a_{2}}{\left|z-a_{2}\right|}\right)^{d_{2}} \ldots\left(\frac{z-a_{m}}{\left|z-a_{m}\right|}\right)^{d_{m}}$. 
After the results of [10], Han and Shafrir, Jerrard, Sandier, Struwe obtained the essential lower bounds for the Dirichlet energy of a unit vector field, see [17,18,21,23].

\section{Preliminary Results}

In this section, we recall some results proved in [15] (see also [16]) useful in the sequel. Let us consider the following quantity, introduced in [15] which will play an important role in our study

$$
I(R, c)=\sup \left\{\int_{1}^{R} \frac{1-f^{2}}{r} d r: \int_{1}^{R} J\left(1-f^{2}\right) r d r \leq c\right\}
$$

for any $R>1$ and $c>0$.

Lemma 1. For every $R>0$ and $c>0$, there exists a maximizer $f_{0}=f_{0}^{(R)}$ in (11) satisfying $0 \leq f_{0}(r) \leq 1$ for every $r$ such that $f_{0}(r)$ is non-decreasing. Moreover, if $r_{0}=r_{0}(c)$ is defined by the equation

$$
c=J(1)\left(\frac{r_{0}^{2}-1}{2}\right),
$$

then there exists $\widetilde{r_{0}}=\widetilde{r_{0}}(c, R) \in\left[1, r_{0}\right]$ such that

$$
f_{0}(r) \begin{cases}=0 & \text { if } r \in[1, R] \text { and } r<\widetilde{r_{0}} \\ >0 & \text { if } r>\widetilde{r_{0}} .\end{cases}
$$

Furthermore

$$
\int_{1}^{R} J\left(1-f_{0}^{2}\right) r d r=c, \forall R>r_{0}
$$

and

$$
j\left(1-f_{0}^{2}\right)=\frac{1}{\lambda r^{2}}, r>\widetilde{r_{0}}
$$

for some $\lambda=\lambda(R, c)>0$.

Moreover, it holds

Lemma 2. There exist two constants $\kappa_{1}>0, \kappa_{2}>0$ such that

$$
\kappa_{1} \min \left(1, \frac{1}{c}\right) \leq \lambda \leq \kappa_{2}\left(1+\frac{1}{c}\right), \quad R \geq r_{0}+1
$$

Actually, the proof of the previous lemma shows that the estimate of $\lambda$ is uniform for $c$ lying in a bounded interval.

Lemma 3. For every $c>1$ there exists a constant $C(c)$ such that for every $c_{1}, c_{2} \in[1 / c, c]$ we have

$$
\left|I\left(R, c_{1}\right)-I\left(R, c_{2}\right)\right| \leq C(c) \quad \forall R \geq 1
$$

In view of Lemma 3 it is natural to set

$$
I(R)=I(R, 1)
$$

and for any fixed $c_{0}>1$ we have

$$
|I(R, c)-I(R)| \leq C\left(c_{0}\right), \quad \forall c \in\left[1 / c_{0}, c_{0}\right] \forall R \geq 1 .
$$

We recall some properties of $I(R)$. 
Lemma 4. We have

$$
I(R)=\frac{1}{2} \int_{\frac{1}{R^{2}}}^{j\left(\eta_{0}\right)} \frac{j^{-1}(t)}{t} d t \quad \forall R \geq 1
$$

In particular,

$$
\lim _{R \rightarrow \infty} \frac{I(R)}{\log R}=0
$$

Moreover for every $\alpha>0$ there exists a constant $C_{1}(\alpha)$ such that

$$
|I(\alpha R)-I(R)| \leq C_{1}(\alpha)
$$

for $R>\max \left(1, \frac{1}{\alpha}\right)$ and $c \in\left(0, c_{0}\right]$.

The next lemma provides an estimate we shall use in the proof of the upper bound in Section 5.1.

Lemma 5. We have

$$
\int_{\mu_{0}}^{R}\left(f_{0}^{\prime}\right)^{2} \leq C, \forall R>\mu_{0}
$$

where $\mu_{0}=\max \left(r_{0}(1), \frac{1}{\sqrt{a j\left(\rho_{0}\right)}}\right)$ being $r_{0}(1)$ and a defined respectively as in Lemmas 1 and 2.

In Theorem 1 we will need a similar functional to that of (11). Hence for $R>1$ and $c>0$ we set

$$
\widetilde{I}(R, c)=\sup \left\{\int_{1}^{R} \frac{1-f^{2}}{r} d r+4 \int_{1}^{R} \frac{\left(1-f^{2}\right)^{2}}{r} d r: \int_{1}^{R} J\left(1-f^{2}\right) r d r \leq c\right\} .
$$

Now, let us recall an important relation between the two functionals (11) and (18).

Lemma 6. There exists a constant $C=C(c)$ such that

$$
|\widetilde{I}(R, c)-I(R, c)| \leq C
$$

for $R>1$.

Lemma 7. There exists a constant $\kappa$ such that for every $c>0, \alpha>0$,

$$
\begin{gathered}
|I(\alpha R, c)-I(R)| \leq \kappa\left(c_{0}, \alpha\right) \\
|\widetilde{I}(\alpha R, c)-I(R)| \leq C_{1}\left(c_{0}, \alpha\right)
\end{gathered}
$$

for $R>\max \left(1, \frac{1}{\alpha}\right)$ and $c \in\left(0, c_{0}\right]$.

The next two propositions, dealing with a lower bound for the energy in a simple annulus and in a more general perforated domain respectively, will play an important role in the proof of our lower bound stated in Section 5.2 (see [15] for details).

Proposition 1. Let $A_{R_{1}, R_{2}}$ denotes the annulus $\left\{R_{1}<|x|<R_{2}\right\}$ and let

$$
u \in C^{1}\left(A_{R_{1}, R_{2}}, \mathbb{C}\right) \cap C\left(\overline{A_{R_{1}, R_{2}}}, \mathbb{C}\right)
$$

satisfy

$$
\operatorname{deg}\left(u, \partial B_{R_{j}}(0)\right)=d, j=1,2
$$




$$
\frac{1}{2} \leq|u| \leq 1 \text { on } A_{R_{1}, R_{2}}
$$

and

$$
\frac{1}{R_{1}^{2}} \int_{A_{R_{1}, R_{2}}} J\left(1-|u|^{2}\right) d x \leq c_{0}
$$

for some constant $c_{0}$. Then there exists a constant $c_{1}$ depending only on $c_{0}$ such that

$$
\int_{A_{R_{1}, R_{2}}}|\nabla u|^{2} d x \geq 2 \pi d^{2}\left(\log \frac{R_{2}}{R_{1}}-I\left(\frac{R_{2}}{R_{1}}\right)\right)-d^{2} c_{1} .
$$

Proposition 2. Let $x_{1}, x_{2}, \ldots, x_{m}$ be $m$ points in $B_{\sigma}(0)$ satisfying

$$
\left|x_{i}-x_{j}\right| \geq 4 \delta, \forall i \neq j \text { and }\left|x_{i}\right|<\frac{\sigma}{4}, \forall i,
$$

with $\delta \leq \frac{\sigma}{32}$. Set $\Omega=B_{\sigma}(0) \backslash \bigcup_{j=1}^{m} B_{\delta}\left(x_{j}\right)$ and let $u$ be a $C^{1}$-map from $\Omega$ into $\mathbb{C}$, which is continuous on $\partial \Omega$ satisfying

$$
\begin{gathered}
\operatorname{deg}\left(u, \partial B_{\sigma}\left(x_{j}\right)\right)=d_{j}, \quad \forall j \\
\frac{1}{2} \leq|u| \leq 1 \operatorname{in} \Omega
\end{gathered}
$$

and

$$
\frac{1}{\delta^{2}} \int_{\Omega} J\left(1-|u|^{2}\right) d x \leq K
$$

Then, denoting $d=\sum_{j=1}^{m} d_{j}$, we have

$$
\int_{\Omega}|\nabla u|^{2} d x \geq 2 \pi|d|\left(\log \frac{\sigma}{\delta}-I\left(\frac{\sigma}{\delta}\right)\right)-C
$$

with $C=C\left(K, m, \sum_{j=1}^{m}\left|d_{j}\right|\right)$.

\section{Lower Bound for the Energy of Unit Vector Fields}

In this section, we will generalize Theorem 4 of [10]. To this aim let $a_{1}, a_{2}, \ldots, a_{m}$ be $m$ points in $B_{R}(0)$ such that

$$
\left|a_{i}-a_{j}\right| \geq 4 R_{0}, \quad \forall i \neq j
$$

and

$$
\left|a_{i}\right| \leq \frac{R}{2}, \quad \forall i
$$

with

$$
R_{0} \leq \frac{R}{4}
$$

Set

$$
\Omega=B_{R}(0) \backslash \bigcup_{j=1}^{m} B_{R_{0}}\left(a_{j}\right)
$$

and let $u$ be a $C^{1}-$ map from $\Omega$ into $C$ which is continuous on $\partial \Omega$.

We suppose that

$$
0<a \leq|u| \leq 1 \text { in } \Omega
$$

and

$$
\frac{1}{R_{0}^{2}} \int_{\Omega} J\left(1-|u|^{2}\right) d x \leq K
$$


for some constants $a$ and $K$.

Let us observe that (23) implies

$$
\operatorname{deg}\left(u, \partial B_{R}\left(a_{j}\right)\right)=d_{j} \quad \forall j
$$

is well defined. Hence, let us denote $d=\sum_{j=1}^{m}\left|d_{j}\right|$ and consider the map

$$
u_{0}(z)=\left(\frac{z-a_{1}}{\left|z-a_{1}\right|}\right)^{d_{1}}\left(\frac{z-a_{2}}{\left|z-a_{2}\right|}\right)^{d_{2}} \ldots\left(\frac{z-a_{m}}{\left|z-a_{m}\right|}\right)^{d_{m}} .
$$

We want to prove the following result

Theorem 2. Let us suppose that (20) $\div(24)$ hold, then we have

$$
\begin{gathered}
\int_{\Omega} p|\nabla u|^{2} d x \geq p_{0} \int_{\Omega}\left|\nabla u_{0}\right|^{2} d x-2 \pi p_{0}\left(\sum_{i=1}^{m} d_{i}^{2}\right) I\left(\frac{R}{R_{0}}\right)+ \\
-2 \pi\left(1-a^{2}\right) p_{0} \sum_{i \neq j}\left|d_{i}\right|\left|d_{j}\right| \log \frac{R}{\left|a_{i}-a_{j}\right|}-C
\end{gathered}
$$

where $C$ is a constant depending only on $p_{0}, a, d, m$ and $K$.

Proof. Let us set $\rho=|u|$ so that $u=\rho e^{i \varphi}$ locally in $\Omega$. Hence we have

$$
|\nabla u|^{2}=|\nabla \rho|^{2}+\rho^{2}|\nabla \varphi|^{2} .
$$

Similarly, we can set $u_{0}=e^{i \varphi_{0}}$ locally in $\Omega$ which implies $\left|\nabla u_{0}\right|=\left|\nabla \varphi_{0}\right|$ and

$$
\nabla \varphi_{0}(z)=\sum_{i=1}^{m} d_{i} \frac{V_{i}(z)}{\left|z-a_{i}\right|}
$$

where

$$
V_{i}(z)=\left(-\frac{y-a_{i}}{\left|z-a_{i}\right|} ; \frac{x-a_{i}}{\left|z-a_{i}\right|}\right)
$$

is the unit vector tangent to the circle of radius $\left|z-a_{i}\right|$ centered at $a_{i}$.

By introducing the function $\psi=\varphi-\varphi_{0}$, we can write $u=\rho u_{0} e^{i \psi}$ and have

$$
|\nabla u|^{2}=|\nabla \rho|^{2}+\rho^{2}\left|\nabla \varphi_{0}+\nabla \psi\right|^{2} .
$$

By (1) and (28) we get

$$
\int_{\Omega} p|\nabla u|^{2} d x \geq p_{0} \int_{\Omega}|\nabla \rho|^{2} d x+p_{0} \int_{\Omega} \rho^{2}\left|\nabla \varphi_{0}\right|^{2} d x+p_{0} \int_{\Omega} \rho^{2}|\nabla \psi|^{2} d x+2 p_{0} \int_{\Omega} \rho^{2} \nabla \varphi_{0} \nabla \psi d x .
$$

By adding and subtracting one in the second and fourth integral and by (23), we get

$$
\begin{aligned}
\int_{\Omega} p|\nabla u|^{2} d x \geq & -p_{0} \int_{\Omega}\left(1-\rho^{2}\right)\left|\nabla \varphi_{0}\right|^{2} d x+p_{0} \int_{\Omega}\left|\nabla \varphi_{0}\right|^{2} d x+p_{0} a^{2} \int_{\Omega}|\nabla \psi|^{2} d x \\
& +2 p_{0} \int_{\Omega}\left(\rho^{2}-1\right) \nabla \varphi_{0} \nabla \psi d x+2 p_{0} \int_{\Omega} \nabla \varphi_{0} \nabla \psi d x
\end{aligned}
$$


Using $2 A B \geq-|A|^{2}-|B|^{2}$, for $A=2\left(\rho^{2}-1\right) \nabla \varphi_{0}$ and $B=\frac{\nabla \psi}{2}$, we can write

$$
\begin{aligned}
\int_{\Omega} p|\nabla u|^{2} d x \geq & -p_{0} \int_{\Omega}\left(1-\rho^{2}\right)\left|\nabla \varphi_{0}\right|^{2} d x+p_{0} \int_{\Omega}\left|\nabla \varphi_{0}\right|^{2} d x+p_{0} a^{2}\|\nabla \psi\|_{2}^{2} \\
& -4 p_{0} \int_{\Omega}\left(\rho^{2}-1\right)^{2}\left|\nabla \varphi_{0}\right|^{2} d x-\frac{p_{0}}{4}\|\nabla \psi\|_{2}^{2}+2 p_{0} \int_{\Omega} \nabla \varphi_{0} \nabla \psi d x .
\end{aligned}
$$

As in Theorem 4 of [8] it holds

$$
\left|\int_{\Omega} \nabla \varphi_{0} \nabla \psi\right| d x \leq C m|d|\|\nabla \psi\|_{2}
$$

for some universal constant $C$, hence (30) becomes

$$
\begin{aligned}
\int_{\Omega} p|\nabla u|^{2} d x \geq & p_{0} \int_{\Omega}\left|\nabla \varphi_{0}\right|^{2} d x-\left[p_{0} \int_{\Omega}\left(1-\rho^{2}\right)\left|\nabla \varphi_{0}\right|^{2} d x+4 p_{0} \int_{\Omega}\left(\rho^{2}-1\right)^{2}\left|\nabla \varphi_{0}\right|^{2} d x\right] \\
& +p_{0}\left(a^{2}-\frac{1}{4}\right)\|\nabla \psi\|_{2}^{2}-2 p_{0} C m|d|\|\nabla \psi\|_{2} .
\end{aligned}
$$

Now let us denote $X=\|\nabla \psi\|_{2}$ and consider the following function

$$
Y=\left(a^{2}-\frac{1}{4}\right) X^{2}-2 C m|d| X .
$$

If $a>\frac{1}{2}$, it reaches its minimum value $Y_{\min }=-\frac{C^{2} m^{2}|d|^{2}}{a^{2}-\frac{1}{4}}$ at $X_{\min }=\frac{C m|d|}{a^{2}-\frac{1}{4}}$. Then we get

$$
\int_{\Omega} p|\nabla u|^{2} d x \geq p_{0} \int_{\Omega}\left|\nabla u_{0}\right|^{2} d x-p_{0}\left[\int_{\Omega}\left(1-\rho^{2}\right)\left|\nabla \varphi_{0}\right|^{2} d x+4 \int_{\Omega}\left(\rho^{2}-1\right)^{2}\left|\nabla \varphi_{0}\right|^{2} d x\right]-C
$$

where $C$ is a constant depending only on $p_{0}, a, d$ and $m$.

Taking into account (11), (18) and (19), in order to get our result, it is enough to estimate the following term

$$
\int_{\Omega}\left(1-\rho^{2}\right)\left|\nabla \varphi_{0}\right|^{2} d x
$$

To this aim let us observe that (27) implies

$$
\left|\nabla \varphi_{0}(z)\right|^{2} \leq \sum_{i=1}^{m} \frac{d_{i}^{2}}{\left|z-a_{i}\right|^{2}}+\sum_{i \neq j} \frac{d_{i} d_{j}}{\left|z-a_{i}\right|\left|z-a_{j}\right|} .
$$

Then (34) can be written as

$$
\begin{aligned}
\int_{\Omega}\left(1-\rho^{2}\right)\left|\nabla \varphi_{0}\right|^{2} d x & =\int_{\Omega}\left(1-\rho^{2}\right)\left[\sum_{i=1}^{m} \frac{d_{i}^{2}}{\left|z-a_{i}\right|^{2}} d z+\sum_{i \neq j} \frac{d_{i} d_{j}}{\left|z-a_{i}\right|\left|z-a_{j}\right|}\right] \\
& \leq \sum_{i=1}^{m} d_{i}^{2} \int_{\Omega} \frac{1-\rho^{2}}{\left|z-a_{i}\right|^{2}} d z+\sum_{i \neq j} d_{i} d_{j} \int_{\Omega} \frac{1-\rho^{2}}{\left|z-a_{i}\right|\left|z-a_{j}\right|} d z \\
& =\sum_{i=1}^{m} d_{i}^{2} A_{i}+B .
\end{aligned}
$$

Let us analyze each term separately. In order to estimate $A_{i}$ for every $i=1, \ldots, m$, let us introduce $\delta_{i}=\operatorname{dist}\left(a_{i}, \partial B_{R}(0)\right)$ and observe that $\frac{R}{2} \leq \delta_{i} \leq R$ as a consequence of (22). 
Therefore for any fixed $i$, by definition (18), it holds

$$
A_{i}=\int_{\Omega} \frac{1-\rho^{2}}{\left|z-a_{i}\right|^{2}} d z \leq \int_{B_{R}(0) \backslash B_{R_{0}}\left(a_{i}\right)} \frac{1-\rho^{2}}{\left|z-a_{i}\right|^{2}} d z \leq 2 \pi I\left(\frac{\delta_{i}}{R_{0}}\right) \leq 2 \pi I\left(\frac{R}{R_{0}}\right)+C
$$

where $C$ depends only on $K$ defined in (24) but is independent of $R, R_{0}$ and $a_{i}$. For the second term, acting as in Theorem 5 of [10] and using (23) we obtain

$$
|B| \leq \sum_{i \neq j}\left|d_{i}\right|\left|d_{j}\right| \int_{\Omega} \frac{1-\rho^{2}}{\left|z-a_{i}\right|\left|z-a_{j}\right|} d z \leq 2 \pi\left(1-a^{2}\right) \sum_{i \neq j}\left|d_{i}\right|\left|d_{j}\right| \log \frac{R}{\left|a_{i}-a_{j}\right|}+C .
$$

where $C$ depends only on $m$ and $d$.

Then by putting together (52) and (37) into (35) we get

$$
\int_{\Omega}\left(1-\rho^{2}\right)\left|\nabla \varphi_{0}\right|^{2} d x \leq 2 \pi\left(\sum_{i=1}^{m} d_{i}^{2}\right) I\left(\frac{R}{R_{0}}\right)+2 \pi\left(1-a^{2}\right) \sum_{i \neq j}\left|d_{i}\right|\left|d_{j}\right| \log \frac{R}{\left|a_{i}-a_{j}\right|}+C .
$$

where $C$ depending on $K, a, m$ and $d$ but does not depend on $R, R_{0}$ and $a_{i}$ for every $i=1, \ldots, m$.

Finally, by (33) and (38) we get (26).

Under the same hypotheses of Theorem 2, as an immediate consequence of (26) and Theorem 5 of [10], we get the following result

Corollary 1. Let us suppose that $(20) \div(24)$ hold, then we have

$$
\begin{aligned}
\int_{\Omega} p|\nabla u|^{2} d x \geq & 2 \pi p_{0}\left(\sum_{i=1}^{m} d_{i}^{2}\right)\left(\log \frac{R}{R_{0}}-I\left(\frac{R}{R_{0}}\right)\right) \\
& +2 \pi p_{0} \sum_{i \neq j}\left(-\left(1-a^{2}\right)\left|d_{i}\right|\left|d_{j}\right|+d_{i} d_{j}\right) \log \frac{R}{\left|a_{i}-a_{j}\right|}-C,
\end{aligned}
$$

where $C$ is a constant depending only on $p_{0}, a, d, m$ and $K$.

Remark 1. If $d_{i} \geq 0$ for $i=1, \ldots$, m then (39) becomes

$$
\int_{\Omega} p|\nabla u|^{2} d x \geq 2 \pi p_{0}\left(\sum_{i=1}^{m} d_{i}^{2}\right)\left(\log \frac{R}{R_{0}}-I\left(\frac{R}{R_{0}}\right)\right)+2 \pi p_{0} a^{2} \sum_{i \neq j} d_{i} d_{j} \log \frac{R}{\left|a_{i}-a_{j}\right|}-C,
$$

where $C$ is a constant depending only on $p_{0}, a, d, m$ and $K$.

\section{Proof of Theorem 1}

Throughout this section, for any subdomain $D$ of $G$ we shall denote

$$
E_{\varepsilon}(u, D)=\int_{D} p|\nabla u|^{2} d x+\frac{1}{\varepsilon^{2}} \int_{D} J\left(1-|u|^{2}\right) d x
$$

and if $D=G$ we simply write $E_{\varepsilon}(u)$. Moreover, similarly to Proposition 1 , we will use the following notation

$$
B_{R_{1}, R_{2}}(b)=\left\{R_{1}<|x-b|<R_{2}\right\}
$$

for the annulus centered in $b$ and with radius $R_{1}$ and $R_{2}$. 
Our main result of this section is the asymptotic behavior of the energy for minimizers which will give (10) of Theorem 1. More precisely we prove the following result

Proposition 3. Assume (5) and (6) hold true. Then for a subsequence $\varepsilon_{n} \rightarrow 0$ we have

$$
\begin{aligned}
E_{\varepsilon_{n}}\left(u_{\varepsilon_{n}}\right)= & 2 \pi p_{0} d \log \frac{1}{\varepsilon_{n}}+2 \pi p_{0}\left(\sum_{k=1}^{N} \frac{d_{k}^{2}-d_{k}}{s_{k}}\right) \log \log \frac{1}{\varepsilon_{n}} \\
& -2 \pi p_{0} d I\left(\frac{1}{\varepsilon_{n}}\left(\log \frac{1}{\varepsilon_{n}}\right)^{-\frac{1}{s_{k}}}\right)+o\left(I\left(\left(\log \frac{1}{\varepsilon_{n}}\right)^{\frac{1}{s_{k}}}\right)\right) .
\end{aligned}
$$

\subsection{An Upper Bound for the Energy}

Let us prove an upper bound for the functional (2).

Proposition 4. Let us suppose that (5) and (6) hold true. Then for a subsequence $\varepsilon_{n} \rightarrow 0$ we have

$$
\begin{aligned}
E_{\varepsilon_{n}}\left(u_{\varepsilon_{n}}\right) \leq & 2 \pi p_{0} d \log \frac{1}{\varepsilon_{n}}+2 \pi p_{0}\left(\Sigma_{k=1}^{N} \frac{d_{k}^{2}-d_{k}}{s_{k}}\right) \log \log \frac{1}{\varepsilon_{n}} \\
& -2 \pi p_{0} d I\left(\frac{1}{\varepsilon_{n}}\left(\log \frac{1}{\varepsilon_{n}}\right)^{-\frac{1}{s_{k}}}\right)+O(1) .
\end{aligned}
$$

Proof. Let $\eta_{0}>0$ satisfy

$$
0<\eta_{0}<\frac{1}{4} \min \left(\min _{i \neq j}\left|\bar{b}_{i}-\bar{b}_{j}\right|, \min _{i=1, \ldots, N} \operatorname{dist}\left(\bar{b}_{i}, \partial G\right)\right)
$$

and fix $k=1, \ldots, N$. Set

$$
T_{\varepsilon_{n}}=\left(\log \frac{1}{\varepsilon_{n}}\right)^{-\frac{1}{s_{k}}} .
$$

We will construct a function $U_{\varepsilon_{n}}(x)$ defined in $\bigcup_{k=1}^{N} B_{\eta_{0}}\left(\bar{b}_{k}\right)$. From this point onwards the proof will develop into three steps.

Step 1. We define $U_{\varepsilon_{n}}(x)=U_{\varepsilon_{n}}^{k}(x)$ on $B_{T_{\varepsilon_{n}}, \eta_{0}}\left(\bar{b}_{k}\right)$ where

$$
U_{\varepsilon_{n}}^{k}(x)=\left(\frac{x-\bar{b}_{k}}{\left|x-\bar{b}_{k}\right|}\right)^{d_{k}}
$$

By following a similar argument as in [3], it is easy to show that

$$
E_{\varepsilon_{n}}\left(U_{\varepsilon_{n}}^{k}, B_{\eta_{0}}\left(\bar{b}_{k}\right) \backslash \overline{B_{T_{\varepsilon_{n}}}\left(\bar{b}_{k}\right)}\right) \leq 2 \pi p_{0} \frac{d_{k}^{2}}{s_{k}} \log \log \frac{1}{\varepsilon_{n}}+O(1)
$$

Step 2. Let us fix $d_{k}$ equidistant points $x_{1}^{n}, x_{2}^{n}, \ldots, x_{d_{k}}^{n}$ on the circle $\partial B_{\frac{T_{\varepsilon}}{2}}\left(\bar{b}_{k}\right)$ and set

$$
A_{\varepsilon_{n}}=B_{T_{\varepsilon_{n}}}\left(\bar{b}_{k},\right) \backslash \bigcup_{j=1}^{d_{k}} B_{\frac{T_{\varepsilon_{n}}}{10 d_{k}}}\left(x_{j}\right) .
$$

We define $U_{\varepsilon_{n}}$ 
as an $S^{1}$-valued map which minimizes the energy $\int_{A_{\varepsilon_{n}}} p|\nabla u|^{2} d x$ among $S^{1}$-valued maps for the boundary data $\left(\frac{x-\bar{b}_{k}}{\left|x-\bar{b}_{k}\right|}\right)^{d_{k}}$ on $\partial B_{T_{\varepsilon_{n}}}\left(\bar{b}_{k},\right)$ and $\frac{x-x_{j}}{\left|x-x_{j}\right|}$ on $\partial B_{\frac{T_{\varepsilon_{n}}}{10 d_{k}}}\left(x_{j},\right), j=1, \ldots, d_{k}$. Clearly we have

$$
E_{\varepsilon_{n}}\left(U_{\varepsilon_{n}}(x), A_{\varepsilon_{n}}\right) \leq C .
$$

Now, let us fix $j \in\left\{1, \ldots, d_{k}\right\}$, let $\vartheta_{j}$ denote a polar coordinate around $x_{j}$ and let $f_{0}(r)$ be a maximizer for $I\left(\frac{1}{\varepsilon_{n}}\left(\log \frac{1}{\varepsilon_{n}}\right)^{-\frac{1}{s_{k}}}\right)$ as given by Lemma 1. Let $\vartheta_{k}$ denote a polar coordinate around $\bar{b}_{k}$, on each $B_{\frac{T_{\varepsilon}}{10 d_{k}}}\left(x_{j}\right)$, according to notation (42), we define $U_{\varepsilon_{n}}(x)=U_{\varepsilon_{n}}^{j, k}(x)$ in $B \frac{T_{\varepsilon_{n}}}{10 d_{k}}\left(\bar{b}_{k}\right)$ where

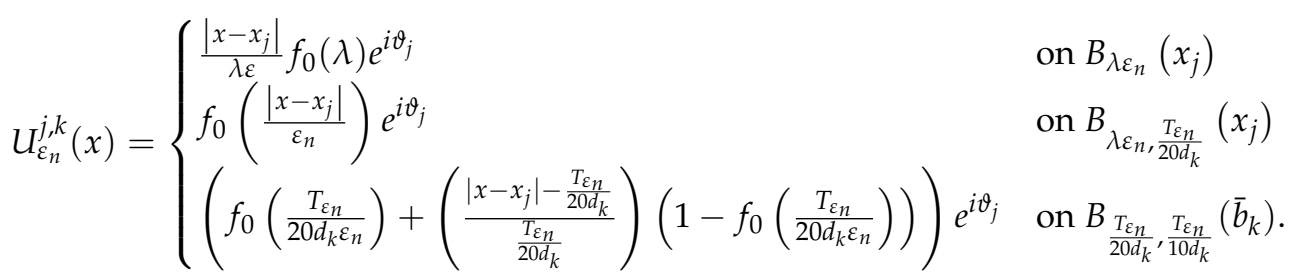

In this step we prove that

$$
\begin{aligned}
E_{\varepsilon_{n}}\left(U_{\varepsilon_{n}}^{j, k}, B_{\frac{T_{\varepsilon}}{10 d_{k}}}\left(x_{j}\right)\right) & \leq-2 \pi p_{0} \frac{1}{s_{k}} \log \log \frac{1}{\varepsilon_{n}}+2 \pi p_{0} \log \frac{1}{\varepsilon_{n}} \\
& -2 \pi p_{0} I\left(\frac{1}{\varepsilon_{n}}\left(\log \frac{1}{\varepsilon_{n}}\right)^{-\frac{1}{s_{k}}}\right)+O(1) .
\end{aligned}
$$

To this aim let us observe that of course we have

$$
E_{\varepsilon_{n}}\left(U_{\varepsilon_{n}}^{j, k}, B_{\lambda \varepsilon_{n}}\left(x_{j}\right)\right)=O(1) .
$$

By putting $U_{\varepsilon_{n}}^{j, k}(x)$ in the energy we obtain

$$
\begin{aligned}
E_{\varepsilon_{n}}\left(U_{\mathcal{\varepsilon}_{n}}^{j, k}, B_{\lambda \varepsilon_{n}, \frac{T_{\varepsilon_{n}}}{20 d_{k}}}\left(x_{j}\right)\right)= & 2 \pi \int_{\lambda \varepsilon_{n}}^{\frac{T_{\varepsilon_{n}}}{20 d_{k}}} p f_{0}^{\prime 2} r d r+\underbrace{2 \pi \int_{\lambda \varepsilon_{n}}^{\frac{T_{\varepsilon_{n}}}{20 d_{k}}} p \frac{f_{0}^{2}}{r} d r}_{(a)}+ \\
& +\frac{2 \pi}{\varepsilon^{2}} \int_{\lambda \varepsilon_{n}}^{\frac{T_{\varepsilon_{n}}}{20 d_{k}}} J\left(1-f_{0}^{2}\right) r d r .
\end{aligned}
$$

By Lemma 5 and (66) we deduce

$$
\int_{\lambda \varepsilon_{n}}^{\frac{T_{\varepsilon}}{20 d_{k}}} p f_{0}^{\prime 2} r d r \leq C
$$

and

$$
\frac{1}{\varepsilon^{2}} \int_{\lambda \varepsilon_{n}}^{\frac{T_{\varepsilon}}{20 d_{k}}} J\left(1-f_{0}^{2}\right) r d r \leq C .
$$

Hence let us split term $(a)$ in (52) in the following way 


$$
(a)=2 \pi \int_{\lambda \varepsilon_{n}}^{\frac{T_{\varepsilon}}{20 d_{k}}} p \frac{f_{0}^{2}}{r} d r=\underbrace{2 \pi \int_{\lambda \varepsilon_{n}}^{\frac{T_{\varepsilon}}{20 d_{k}}}\left(p-p_{0}\right) \frac{f_{0}^{2}}{r} d r}_{(1)}+\underbrace{2 \pi p_{0} \int_{\lambda \varepsilon_{n}}^{\frac{T_{\varepsilon}}{20 d_{k}}} \frac{f_{0}^{2}}{r} d r}_{(2)} .
$$

Let us observe that

$$
\left|x-\bar{b}_{k}\right|^{s_{k}} \leq 2^{s_{k}}\left(\left|x-x_{j}\right|^{s_{k}}+\left|x_{j}-\bar{b}_{k}\right|^{s_{k}}\right) \quad \forall j \in\left\{1, \ldots, d_{k}\right\},
$$

hence, by (6) we have

$$
\begin{aligned}
(1) & \leq \frac{2^{s_{k}+1}}{\left(20 d_{k}\right)^{s_{k}}} \pi \beta_{k}\left(\log \frac{1}{\varepsilon_{n}}\right)^{-1} \int_{\lambda \varepsilon}^{\frac{T_{\varepsilon}}{20 d_{k}}} \frac{f_{0}^{2}}{r} d r+2 \pi \beta_{k}\left(\log \frac{1}{\varepsilon_{n}}\right)^{-1} \int_{\lambda \varepsilon_{n}}^{\frac{T_{\varepsilon_{n}}}{20 d_{k}}} \frac{f_{0}^{2}}{r} d r \\
& =-2 \pi \beta_{k}\left(\frac{1}{10^{s_{k}} d_{k}^{s_{k}}}+1\right)\left(\log \frac{1}{\varepsilon_{n}}\right)^{-1} \int_{\lambda \varepsilon_{n}}^{\frac{T_{\varepsilon}}{20 d_{k}}} \frac{1-f_{0}^{2}}{r} d r+ \\
& +2 \pi \beta_{k}\left(\frac{1}{10^{s_{k}} d_{k}^{s_{k}}}+1\right)\left(\log \frac{1}{\varepsilon_{n}}\right)^{-1} \int_{\lambda \varepsilon_{n}}^{\frac{T_{\varepsilon_{n}}}{20 d_{k}}} \frac{d r}{r} .
\end{aligned}
$$

By Lemma 1 and Lemma 7

$$
\begin{aligned}
(1) \leq & -2 \pi \beta_{k}\left(\frac{1}{10^{s_{k}} d_{k}^{s_{k}}}+1\right)\left(\log \frac{1}{\varepsilon_{n}}\right)^{-1} I\left(\frac{1}{\varepsilon_{n}}\left(\log \frac{1}{\varepsilon_{n}}\right)^{-\frac{1}{s_{k}}}\right) \\
& +2 \pi \beta_{k}\left(\frac{1}{10^{s_{k}} d_{k}^{s_{k}}}+1\right)\left(\log \frac{1}{\varepsilon_{n}}\right)^{-1}\left[-\frac{1}{s_{k}} \log \log \frac{1}{\varepsilon_{n}}+\log \frac{1}{\lambda \varepsilon_{n}}\right]+O(1) \\
= & -2 \pi \beta_{k}\left(\frac{1}{10^{s_{k}} d_{k}^{s_{k}}}+1\right)\left(\log \frac{1}{\varepsilon_{n}}\right)^{-1} I\left(\frac{1}{\varepsilon_{n}}\left(\log \frac{1}{\varepsilon_{n}}\right)^{-\frac{1}{s_{k}}}\right) \\
& -2 \frac{\pi \beta_{k}}{s_{k}}\left(\frac{1}{10^{s_{k}} d_{k}^{s_{k}}}+1\right)\left(\log \frac{1}{\varepsilon_{n}}\right)^{-1} \log \log \frac{1}{\varepsilon_{n}} \\
& +2 \pi \beta_{k}\left(\frac{1}{10^{s_{k}} d_{k}^{s_{k}}}+1\right)\left(1-\log \lambda\left(\log \frac{1}{\varepsilon_{n}}\right)^{-1}\right)+O(1) .
\end{aligned}
$$

Let us observe that

$$
\lim _{n \rightarrow+\infty}\left(\log \frac{1}{\varepsilon_{n}}\right)^{-1} \log \log \frac{1}{\varepsilon_{n}}=0
$$

and again by (16) that

$$
\lim _{n \rightarrow+\infty}\left(\log \frac{1}{\varepsilon_{n}}\right)^{-1} I\left(\frac{1}{\varepsilon_{n}}\left(\log \frac{1}{\varepsilon_{n}}\right)^{-\frac{1}{s_{k}}}\right)=0 .
$$

Then we can conclude

$$
(1) \leq O(1) \text {. }
$$

Now let us consider the second term in the right hand side of (55) 


$$
\begin{aligned}
(2) & =2 \pi p_{0} \int_{\lambda \varepsilon_{n}}^{\frac{T_{\varepsilon_{n}}}{20 d_{k}}} \frac{f_{0}^{2}}{r} d r=-2 \pi p_{0} \int_{\lambda \varepsilon_{n}}^{\frac{T_{\varepsilon_{n}}}{20 d_{k}}} \frac{1-f_{0}^{2}}{r} d r+2 \pi p_{0} \int_{\lambda \varepsilon_{n}}^{\frac{T_{\varepsilon}}{20 d_{k}}} \frac{d r}{r} \\
& =-2 \pi p_{0} I\left(\frac{1}{\varepsilon_{n}}\left(\log \frac{1}{\varepsilon_{n}}\right)^{-\frac{1}{s_{k}}}\right)+2 \pi p_{0}\left(-\frac{1}{s_{k}} \log \log \frac{1}{\varepsilon_{n}}+\log \frac{1}{\lambda \varepsilon_{n}}\right)+O(1) \\
& =-2 \pi p_{0} I\left(\frac{1}{\varepsilon_{n}}\left(\log \frac{1}{\varepsilon_{n}}\right)^{-\frac{1}{s_{k}}}\right)-2 \pi p_{0} \frac{1}{s_{k}} \log \log \frac{1}{\varepsilon_{n}}+2 \pi p_{0} \log \frac{1}{\varepsilon_{n}}+O(1) .
\end{aligned}
$$

By collecting together, we get

$$
(a)=(1)+(2) \leq-2 \pi p_{0} I\left(\frac{1}{\varepsilon_{n}}\left(\log \frac{1}{\varepsilon_{n}}\right)^{-\frac{1}{s_{k}}}\right)-2 \pi p_{0} \frac{1}{s_{k}} \log \log \frac{1}{\varepsilon_{n}}+2 \pi p_{0} \log \frac{1}{\varepsilon_{n}}+O(1) .
$$

Let us observe that (50) will follows from (51), (53), (54) and (57) once we prove that

$$
E_{\varepsilon_{n}}\left(U_{\varepsilon_{n}}^{j, k}, B_{\frac{T_{\varepsilon_{n}}}{20 d_{k}}, \frac{T_{\varepsilon_{n}}}{10 d_{k}}}\left(x_{j}\right)\right) \leq C .
$$

To verify (58) we write,

$$
U_{\varepsilon_{n}}^{j, k}\left(x_{j}+r e^{i \vartheta_{j}}\right)=z(r) e^{i \vartheta_{j}} \text { on } B_{\frac{T_{\varepsilon}}{20 d_{k}}, \frac{T_{\varepsilon}}{10 d_{k}}}\left(x_{j}\right)
$$

where

$$
z(r)=f_{0}\left(\frac{T_{\varepsilon_{n}}}{20 d_{k} \varepsilon_{n}}\right)+\left(\frac{r-\frac{T_{\varepsilon_{n}}}{20 d_{k}}}{\frac{T_{\varepsilon_{n}}}{20 d_{k}}}\right)\left(1-f_{0}\left(\frac{T_{\varepsilon_{n}}}{20 d_{k} \varepsilon_{n}}\right)\right) .
$$

Acting as in Proposition 3.1 in [15], by the properties of $f_{0}$ of Lemma 1 and as $T_{\varepsilon_{n}}$ go to zero when $\varepsilon_{n}$ tends to zero, we compute

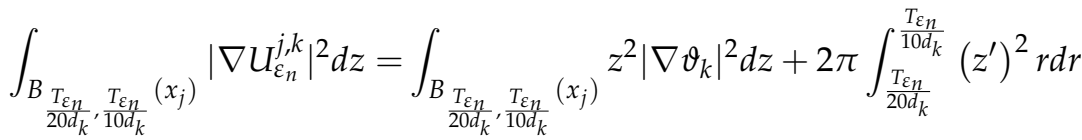

$$
\begin{aligned}
& =O(1)+2 \pi\left(\frac{1-f_{0}\left(\frac{T_{\varepsilon_{n}}}{20 d_{k} \varepsilon_{n}}\right)}{\eta_{0}}\right)^{2} \int_{\frac{\tau_{n}}{20 d_{k}}}^{\frac{T_{\varepsilon}}{10 d_{k}}} r d r \leq C .
\end{aligned}
$$

About the second term of the energy, using the inequality $J(t) \leq t j(t)$, Lemma 1 and Lemma 2 , we obtain

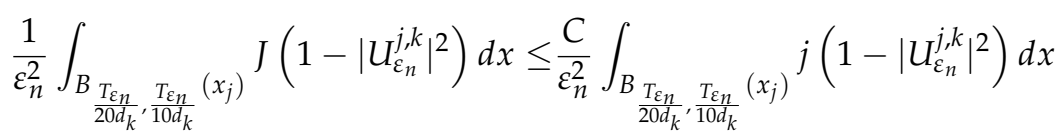

$$
\begin{aligned}
& \leq \frac{C}{\varepsilon_{n}^{2}} j\left(1-f_{0}^{2}\left(\frac{T_{\varepsilon_{n}}}{20 d_{k} \varepsilon_{n}}\right)\right)\left(\frac{T_{\varepsilon_{n}}^{2}}{100 d_{k}^{2}}-\frac{T_{\varepsilon_{n}}^{2}}{400 d_{k}^{2}}\right) \\
& =\frac{C}{\varepsilon_{n}^{2}} \frac{3}{\lambda\left(\frac{T_{\varepsilon_{n}}}{20 d_{k} \varepsilon_{n}}\right)^{2}} \frac{T_{\varepsilon_{n}}^{2}}{400}=O(1) \text {. }
\end{aligned}
$$

Hence by (60) and (61) we get (58).

Finally, by (51), (53), (54), (57) and (58) we can write

$$
E_{\varepsilon_{n}}\left(U_{\varepsilon_{n}}^{j, k}, B \frac{T_{\varepsilon_{n}}}{10 d_{k}}\left(x_{j}\right)\right) \leq-2 \pi p_{0} \frac{1}{s_{k}} \log \log \frac{1}{\varepsilon_{n}}+2 \pi p_{0} \log \frac{1}{\varepsilon_{n}}-2 \pi p_{0} I\left(\frac{1}{\varepsilon_{n}}\left(\log \frac{1}{\varepsilon_{n}}\right)^{-\frac{1}{s_{k}}}\right)+O(1) .
$$


Step 3. We define the function $U_{\varepsilon_{n}}$ in $\bigcup_{j=1}^{d_{k}} B_{T_{\varepsilon_{n}}}\left(x_{j}\right)$ such that

$$
U_{\varepsilon_{n}}^{k}(x)=U_{\varepsilon_{n}}^{j, k}(x) \quad \text { if } \quad x \in B_{T_{\varepsilon_{n}}}\left(x_{j}\right) .
$$

As the discs centered in $x_{j}$ are disjoint and as they are exactly $d_{k}$ discs we get

$$
\begin{aligned}
E\left(U_{\varepsilon_{n}}^{k}(x), \bigcup_{j=1}^{d_{k}} B_{T_{\varepsilon_{n}}}\left(x_{j}\right)\right) \leq & -2 \pi p_{0} d_{k} I\left(\frac{1}{\varepsilon_{n}}\left(\log \frac{1}{\varepsilon_{n}}\right)^{-\frac{1}{s_{k}}}\right)-2 \pi p_{0} \frac{d_{k}}{s_{k}} \log \log \frac{1}{\varepsilon_{n}} \\
& +2 \pi p_{0} d_{k} \log \frac{1}{\varepsilon_{n}}+O(1) .
\end{aligned}
$$

By (47), (48) and (63) we have

$$
\begin{aligned}
E_{\varepsilon_{n}}\left(U_{\varepsilon_{n}}^{k}, B_{\eta_{0}}\left(\bar{b}_{k}\right)\right) \leq & 2 \pi p_{0} \frac{d_{k}^{2}}{s_{k}} \log \log \frac{1}{\varepsilon_{n}}-2 \pi p_{0} d_{k} I\left(\frac{1}{\varepsilon_{n}}\left(\log \frac{1}{\varepsilon_{n}}\right)^{-\frac{1}{s_{k}}}\right) \\
& -2 \pi p_{0} \frac{d_{k}}{s_{k}} \log \log \frac{1}{\varepsilon_{n}}+2 \pi p_{0} d_{k} \log \frac{1}{\varepsilon_{n}}+O(1) .
\end{aligned}
$$

Finally, we pose $U_{\mathcal{E}_{n}}(x)=w$ on $G \backslash \bigcup_{k=1}^{N} \overline{B_{\eta_{0}}\left(\bar{b}_{k}\right)}$ where $w$ is any $S^{1}$-valued map of class $C^{1}$ on this domain which equals $g$ on $\partial G$ and $\left(\frac{x-\bar{b}_{k}}{\left|x-\bar{b}_{k}\right|}\right)^{d_{k}}$ on $\partial B_{\eta_{0}}\left(\bar{b}_{k}\right)$ for $k=1, \ldots, N$. Then $U_{\varepsilon_{n}} \in$ $H_{g}^{1}(G, \mathbf{C})$ and we get

$$
\begin{aligned}
E_{\varepsilon_{n}}\left(u_{\varepsilon_{n}}\right) \leq E_{\varepsilon_{n}}\left(U_{\varepsilon_{n}}\right) \leq & 2 \pi p_{0} d \log \frac{1}{\varepsilon_{n}}+2 \pi p_{0} \Sigma_{k=1}^{N} \frac{d_{k}^{2}-d_{k}}{s_{k}} \log \log \frac{1}{\varepsilon_{n}} \\
& -2 \pi p_{0} d I\left(\frac{1}{\varepsilon_{n}}\left(\log \frac{1}{\varepsilon_{n}}\right)^{-\frac{1}{s_{k}}}\right)+O(1)
\end{aligned}
$$

which is (44).

\subsection{A Lower Bound for the Energy}

When $G$ is star shaped, using a Pohozaev identity, we obtain

$$
\frac{1}{\varepsilon^{2}} \int_{G} J\left(1-\left|u_{\varepsilon}\right|^{2}\right) d x \leq C_{0}, \forall \varepsilon>0 .
$$

By following the same arguments of Lemmas 3.1 and 3.2 in [15] we get

$$
\left\|u_{\varepsilon}\right\|_{L^{\infty}(G)} \leq 1 \text { and }\left\|\nabla u_{\varepsilon}\right\|_{L^{\infty}(G)} \leq \frac{C}{\varepsilon} .
$$

Using the construction in [6] we know that there exist $\lambda>0$ and a collection of balls $\left\{B_{\lambda \varepsilon}\left(y_{j}^{\varepsilon}\right)\right\}_{j \in J}$ such that

$$
\begin{gathered}
\left\{x \in \bar{G}:\left|u_{\varepsilon}(x)\right| \leq \frac{3}{4}\right\} \subset \bigcup_{j \in J} B_{\lambda \varepsilon}\left(y_{j}^{\varepsilon}\right), \\
\left|y_{i}^{\varepsilon}-y_{j}^{\varepsilon}\right| \geq 8 \lambda \varepsilon \forall i, j \in J, i \neq j
\end{gathered}
$$

and

$$
\operatorname{card} \mathrm{J} \leq \mathrm{N}_{\mathrm{b}}
$$


By construction, the degrees

$$
v_{j}=\operatorname{deg}\left(u_{\varepsilon}, \partial B_{\lambda \varepsilon}\left(y_{j}^{\varepsilon}\right)\right), j \in J
$$

are well defined. Given any subsequence $\varepsilon_{n} \rightarrow 0$ we may extract a subsequence (still denoted by $\varepsilon_{n}$ ) such that

$$
\operatorname{card} J_{\varepsilon_{n}}=\text { const }=\mathrm{N}_{1}
$$

and

$$
y_{j}=y_{j}^{\varepsilon_{n}} \rightarrow l_{j} \in \bar{G}, j=1, \ldots, N_{1} .
$$

Let $\underline{b}_{1}, \underline{b}_{2}, \ldots, \underline{b}_{N_{2}}$ be the distinct points among the $\left\{l_{j}\right\}_{j=1}^{N_{1}}$ and set

$$
I_{k}=\left\{j \in\left\{1, \ldots, N_{1}\right\} ; y_{j}^{\varepsilon_{n}} \rightarrow \underline{b}_{k}\right\}, k=1, \ldots, N_{2} .
$$

Denoting by $d_{k}=\sum_{j \in I_{k}} v_{j}$ for every $k=1, \ldots, N_{2}$, we clearly have and $\sum_{k=1}^{N_{2}} d_{k}=d$. By following the same arguments as in [3], thanks to the previous upper bound, applied to $\bar{b}_{k}=\underline{b}_{k}$, and Proposition 2, we get

$$
d_{k}>0 \text { for every } k=1, \ldots, N_{2}
$$

and

$$
\underline{b}_{k} \in \Lambda=p^{-1}\left(p_{0}\right) \text { for every } k=1, \ldots, N_{2} .
$$

Hence, having in mind (5), in the following we can set $N_{2}=N$ and $\underline{b}_{k}=b_{k}$. Moreover, acting as in [3], Lemma 2.1 by Propositions 1 and 2 , we get $v_{j}=+1$ for every $j \in I_{k}$.

Let $\eta$ satisfy

$$
0<\eta<\frac{1}{2} \min \left(\min _{i \neq j}\left|b_{i}-b_{j}\right|,_{i=1, \ldots, N_{2}} \operatorname{dist}\left(b_{i}, \partial G\right)\right) .
$$

and take $T_{\mathcal{E}_{n}}$ as in (45). We now are able to prove the following lower bound:

Proposition 5. Assume $G$ is star shaped and (5) and (6) hold true. Then we have, for a subsequence $\varepsilon_{n} \rightarrow 0$

$$
\begin{aligned}
E_{\varepsilon_{n}}\left(u_{\varepsilon_{n}}\right) \geq & 2 \pi p_{0} d \log \frac{1}{\varepsilon_{n}}+2 \pi p_{0} \sum_{k=1}^{N} \frac{d_{k}^{2}-d_{k}}{s_{k}} \log \log \frac{1}{\varepsilon_{n}}-2 \pi p_{0} d I\left(\frac{1}{\varepsilon_{n}}\left(\log \frac{1}{\varepsilon_{n}}\right)^{-\frac{1}{s_{k}}}\right) \\
& -2 \pi p_{0} \sum_{k=1}^{N} d_{k}^{2} I\left(\left(\log \frac{1}{\varepsilon_{n}}\right)^{\frac{1}{s_{k}}}\right)+\frac{9}{8} \pi p_{0} \sum_{k=1}^{N} \sum_{i \neq j} \log \frac{\left(\log \frac{1}{\varepsilon_{n}}\right)^{-\frac{1}{s_{k}}}}{\left|y_{i}-y_{j}\right|}+O(1),
\end{aligned}
$$

where the points $y_{i}$ and $y_{j}, i, j \in I_{k}$, are as in (69).

Proof. The proof develops into two steps.

Step 1. By following a similar argument as in [3], at first we prove

$$
\max _{i \in I_{k}}\left|b_{k}-y_{i}\right|=R_{n} \sim\left|\log \varepsilon_{n}\right|^{-\frac{1}{s_{k}}}
$$

for every $k=1, \ldots, N_{2}$ with $\left|I_{k}\right|=d_{k}>1$.

We know that $B_{\eta}\left(b_{k}\right)$ contains exactly $d_{k}$ bad discs $B_{\lambda \varepsilon_{n}}\left(y_{i}\right)$, such that for every $\alpha \in(0,1)$

$$
\left|y_{i}-y_{j}\right|>\varepsilon_{n}^{\alpha} \quad \forall i \neq j
$$


For any fixed $\alpha \in(0,1)$, we have

$$
\begin{aligned}
E\left(u_{\varepsilon_{n}}, B_{\eta}\left(b_{k}\right)\right) \geq & E\left(u_{\varepsilon_{n}}, B_{2 R_{n}, \eta}\left(b_{k}\right)\right)+E\left(u_{\varepsilon}, B_{2 R_{n}}\left(b_{k}\right) \backslash \bigcup_{i \in I_{k}} B_{\varepsilon_{n}^{\alpha}}\left(y_{i}\right)\right) \\
& +E\left(u_{\varepsilon_{n}}, \bigcup_{i \in I_{k}} B_{\lambda \varepsilon_{n}, \varepsilon_{n}^{\alpha}}\left(y_{i}\right)\right)=(a)+(b)+(c) .
\end{aligned}
$$

Taking into account (66), by Proposition 1 , there exist two constants $C_{1}$ and $C_{3}$ depending only on $C_{0}$ and a constant $C_{2}$ depending on $C_{0}$ and $d_{k}$, such that

$$
\begin{gathered}
(a) \geq 2 \pi d_{k}^{2} p_{0}\left[\log \frac{\eta}{2 R_{n}}-I\left(\frac{\eta}{2 R_{n}}\right)\right]-d_{k}^{2} C_{1}, \\
(b) \geq 2 \pi d_{k} p_{0}\left[\log \frac{2 R_{n}}{\varepsilon_{n}^{\alpha}}-I\left(\frac{2 R_{n}}{\varepsilon_{n}^{\alpha}}\right)\right]-C_{2}
\end{gathered}
$$

and

$$
(c) \geq 2 \pi\left(d_{k}-1\right) p_{0}\left[\log \frac{\varepsilon_{n}^{\alpha}}{\lambda \varepsilon_{n}}-I\left(\frac{\varepsilon_{n}^{\alpha}}{\lambda \varepsilon_{n}}\right)\right]+2 \pi\left(p_{0}+\alpha_{k} \frac{R_{k}^{s_{k}}}{4}\right)\left[\log \frac{\varepsilon_{n}^{\kappa}}{\lambda \varepsilon_{n}}-I\left(\frac{\varepsilon_{n}^{\alpha}}{\lambda \varepsilon_{n}}\right)\right]-C_{3} .
$$

Let us denote

$$
f\left(R_{n}\right)=2 \pi p_{0} d_{k} \log \frac{1}{\varepsilon_{n}}+2 \pi p_{0}\left(d_{k}^{2}-d_{k}\right) \log \frac{1}{R_{n}}+\frac{\pi}{2} \alpha_{k}(1-\alpha) R_{n}^{s_{k}} \log \frac{1}{\varepsilon_{n}}
$$

and

$$
g\left(R_{n}\right)=2 \pi d_{k}^{2} p_{0} I\left(\frac{1}{R_{n}}\right)+2 \pi d_{k} p_{0} I\left(\frac{R_{n}}{\varepsilon_{n}^{\alpha}}\right)+2 \pi\left(p_{0}+\alpha_{k} \frac{R_{n}^{s_{k}}}{4}\right) I\left(\frac{1}{\varepsilon_{n}^{1-\alpha}}\right)+C_{4} .
$$

where $C_{4}$ is a constant depending only on $C_{0}$ and $d_{k}$. Then

$$
E\left(u_{\varepsilon_{n}}, B_{\eta}\left(b_{k}\right)\right) \geq f\left(R_{n}\right)-g\left(R_{n}\right)-C_{4} .
$$

Now let us observe that for $n$ large enough, we get

$$
\frac{\eta}{2 R_{n}} \geq 1
$$

since $R_{n}$ tends to 0 . Moreover, by (75) it holds

$$
\varepsilon_{n}^{\alpha}<\left|y_{i}-y_{j}\right| \leq\left|y_{i}-b_{k}\right|+\left|y_{j}-b_{k}\right| \leq 2 R_{n} \quad \forall i \neq j .
$$

Hence we get

$$
\frac{\varepsilon_{n}^{\alpha}}{2} \leq R_{n} \leq \frac{\eta}{2}
$$

Let us pose $R_{n}=c_{n}\left(\log \frac{1}{\varepsilon_{n}}\right)^{-\frac{1}{s_{k}}}$ and consider the following difference 


$$
\begin{aligned}
& {\left[f\left(R_{n}\right)-g\left(R_{n}\right)\right]-\left[f\left(\left(\log \frac{1}{\varepsilon_{n}}\right)^{-\frac{1}{s_{k}}}\right)-g\left(\left(\log \frac{1}{\varepsilon_{n}}\right)^{-\frac{1}{s_{k}}}\right)\right]=} \\
& \underbrace{\left[f\left(R_{n}\right)-f\left(\left(\log \frac{1}{\varepsilon_{n}}\right)^{-\frac{1}{s_{k}}}\right)\right]}_{(1)}+\underbrace{\left[g\left(\left(\log \frac{1}{\varepsilon_{n}}\right)^{-\frac{1}{s_{k}}}\right)-g\left(R_{n}\right)\right]}_{(2)} .
\end{aligned}
$$

By (80) and (81) we get

$$
(1)=2 \pi p_{0}\left(d_{k}^{2}-d_{k}\right) \log \frac{1}{c_{n}}+\frac{\pi}{2} \alpha_{k}(1-\alpha)\left(c_{n}^{s_{k}}-1\right)
$$

and

$$
\begin{aligned}
(2)= & 2 \pi d_{k}^{2} p_{0}\left(I\left(\left(\log \frac{1}{\varepsilon_{n}}\right)^{\frac{1}{s_{k}}}\right)-I\left(\frac{1}{c_{n}}\left(\log \frac{1}{\varepsilon_{n}}\right)^{\frac{1}{s_{k}}}\right)\right)+ \\
& +2 \pi p_{0} d_{k}\left(I\left(\frac{1}{\varepsilon_{n}^{\alpha}}\left(\log \frac{1}{\varepsilon_{n}}\right)^{-\frac{1}{s_{k}}}\right)-I\left(\frac{c_{n}}{\varepsilon_{n}^{\alpha}}\left(\log \frac{1}{\varepsilon_{n}}\right)^{-\frac{1}{s_{k}}}\right)\right)+ \\
& +\frac{\pi \alpha_{k}\left(1-c_{n}^{s_{k}}\right)}{2}\left(\log \frac{1}{\varepsilon_{n}}\right)^{-1} I\left(\frac{1}{\varepsilon_{n}^{1-\alpha}}\right) .
\end{aligned}
$$

Let us consider the case $c_{n}>1$. Therefore we have

$$
R_{n}>\left(\log \frac{1}{\varepsilon_{n}}\right)^{-\frac{1}{s_{k}}} .
$$

By (15), (86) and as the functions $j^{-1}$ and $I$ are increasing, we get

$$
\begin{aligned}
& (2) \geq-2 \pi p_{0} d_{k} \int_{\frac{\varepsilon_{n}}{R_{n}^{2}}}^{\left(\log \frac{1}{\varepsilon_{n}}\right)^{\frac{2}{s_{k}}} \varepsilon_{n}^{2 \alpha}} \frac{j^{-1}(t)}{t} d t+\frac{\pi \alpha_{k}\left(1-c_{n}^{s_{k}}\right)}{2}\left(\log \frac{1}{\varepsilon_{n}}\right)^{-1} I\left(\frac{1}{\varepsilon_{n}^{1-\alpha}}\right) \\
& \geq-2 \pi p_{0} d_{k} j^{-1}\left(\left(\log \frac{1}{\varepsilon_{n}}\right)^{\frac{2}{s_{k}}} \varepsilon_{n}^{2 \alpha}\right) \log c_{n}^{2}+\frac{\pi \alpha_{k}\left(1-c_{n}^{s_{k}}\right)}{2}\left(\log \frac{1}{\varepsilon_{n}}\right)^{-1} I\left(\frac{1}{\varepsilon_{n}^{1-\alpha}}\right) .
\end{aligned}
$$

Since

$$
\lim _{n \rightarrow+\infty}\left(\log \frac{1}{\varepsilon_{n}}\right)^{\frac{2}{s_{k}}} \varepsilon_{n}^{2 \alpha}=0
$$

and by (16)

$$
\lim _{n \rightarrow+\infty}\left(\log \frac{1}{\varepsilon_{n}}\right)^{-1} I\left(\frac{1}{\varepsilon_{n}^{1-\alpha}}\right)=0,
$$

by regularity of function $j^{-1}$ and as $j^{-1}(0)=0$, there exists $n_{0}$ such that for $n \geq n_{0}$ we have

$$
\text { (2) } \geq 2 \delta \pi p_{0} d_{k} \log \frac{1}{c_{n}}+\frac{\pi}{2} \alpha_{k}\left(1-c_{n}^{s_{k}}\right) \gamma .
$$

Then, by denoting

$$
h\left(R_{n}\right)=f\left(R_{n}\right)-g\left(R_{n}\right)
$$


by (84) and (88) and choosing $\delta=\frac{1}{2}$ and $\gamma=\frac{1-\alpha}{2}$, we get

$$
h\left(R_{n}\right)-h\left(\left(\log \frac{1}{\varepsilon_{n}}\right)^{-\frac{1}{s_{k}}}\right) \geq 2 \pi p_{0}\left(d_{k}^{2}-\frac{d_{k}}{2}\right) \log \frac{1}{c_{n}}+\frac{\pi}{8} \alpha_{k}(1-\alpha)\left(c_{n}^{s_{k}}-1\right) .
$$

Hence we get

$$
h\left(R_{n}\right)-h\left(\left(\log \frac{1}{\varepsilon_{n}}\right)^{-\frac{1}{s_{k}}}\right) \rightarrow+\infty \text { as } c_{n} \rightarrow+\infty .
$$

Now let us suppose there exists a subsequence $\left(c_{n_{k}}\right)_{k}$, still denoted by $\left(c_{n}\right)$, such that $c_{n}<1$. Up to a subsequence we have

$$
R_{n}<\left(\log \frac{1}{\varepsilon_{n}}\right)^{-\frac{1}{s_{k}}}
$$

By (15), (91) and as the functions $j^{-1}$ and $I$ are increasing, we get

$$
\begin{aligned}
(2) & \geq-2 \pi p_{0} d_{k}^{2} \int_{R_{n}^{2}}^{\left(\log \frac{1}{\varepsilon_{n}}\right)^{-\frac{2}{s_{k}}}} \frac{j^{-1}(t)}{t} d t+\frac{\pi}{2} \alpha_{k}\left(1-c_{n}^{s_{k}}\right)\left(\log \frac{1}{\varepsilon_{n}}\right)^{-1} I\left(\frac{1}{\varepsilon_{n}^{1-\alpha}}\right) \\
& \geq-2 \pi p_{0} d_{k}^{2} j^{-1}\left(\left(\log \frac{1}{\varepsilon_{n}}\right)^{-\frac{2}{s_{k}}}\right) \log \frac{1}{c_{n}^{2}}+\frac{\pi}{2} \alpha_{k}\left(1-c_{n}^{s_{k}}\right)\left(\log \frac{1}{\varepsilon_{n}}\right)^{-1} I\left(\frac{1}{\varepsilon_{n}^{1-\alpha}}\right) .
\end{aligned}
$$

Since

$$
\lim _{n \rightarrow+\infty}\left(\log \frac{1}{\varepsilon_{n}}\right)^{-\frac{2}{s_{k}}}=0
$$

and by (16)

$$
\lim _{n \rightarrow+\infty}\left(\log \frac{1}{\varepsilon_{n}}\right)^{-1} I\left(\frac{1}{\varepsilon_{n}^{1-\alpha}}\right)=0,
$$

similarly to the previous case, by regularity of function $j^{-1}$ and as $j^{-1}(0)=0$ there exists $n_{0}$ such that for $n \geq n_{0}$ we have

$$
(2) \geq-2 \delta \pi p_{0} d_{k}^{2} \log \frac{1}{c_{n}}+\frac{\pi}{2} \alpha_{k}\left(1-c_{n}^{s_{k}}\right) \gamma \geq-2 \delta \pi p_{0} d_{k}^{2} \log \frac{1}{c_{n}} .
$$

Then, by denoting

$$
h\left(R_{n}\right)=f\left(R_{n}\right)-g\left(R_{n}\right),
$$

by (84) and (92) we get

$$
h\left(R_{n}\right)-h\left(\left(\log \frac{1}{\varepsilon_{n}}\right)^{-\frac{1}{s_{k}}}\right) \geq 2 \pi p_{0}\left(d_{k}^{2}-d_{k}-\delta d_{k}^{2}\right) \log \frac{1}{c_{n}}+\frac{\pi}{2} \alpha_{k}(1-\alpha)\left(c_{n}^{s_{k}}-1\right) .
$$

Let us choose $\delta>0$ such that $d_{k}^{2}-d_{k}-\delta d_{k}^{2}>1$ or equivalently $\delta<1-\frac{1+d_{k}}{d_{k}^{2}}$. This is possible as $d_{k}>1$ and then $1-\frac{1+d_{k}}{d_{k}^{2}}>0$. For this choice it holds

$$
h\left(R_{n}\right)-h\left(\left(\log \frac{1}{\varepsilon_{n}}\right)^{-\frac{1}{s_{k}}}\right) \rightarrow+\infty \text { as } \frac{1}{c_{n}} \rightarrow+\infty .
$$


By (90) and (93), in both cases we can conclude as in [3]

$$
h\left(R_{n}\right)-h\left(\left(\log \frac{1}{\varepsilon_{n}}\right)^{-\frac{1}{s_{k}}}\right) \rightarrow+\infty \text { as } \max \left(c_{n}, \frac{1}{c_{n}}\right) \rightarrow+\infty .
$$

By (82) we get

$$
h\left(R_{n}\right)-h\left(\left(\log \frac{1}{\varepsilon_{n}}\right)^{-\frac{1}{s_{k}}}\right) \leq E\left(u_{\varepsilon_{n}}, B_{\eta}\left(b_{k}\right)\right)+C_{4}-h\left(\left(\log \frac{1}{\varepsilon_{n}}\right)^{-\frac{1}{s_{k}}}\right) .
$$

We know that $\bar{b}_{k}=b_{j}$ for some $j \in\{1, \ldots, N\}$. Hence by using the upper bound (44) of Proposition 4, taking into account (80), (81) and (89), since $\alpha<1$, we obtain

$$
\begin{gathered}
h\left(R_{n}\right)-h\left(\left(\log \frac{1}{\varepsilon_{n}}\right)^{-\frac{1}{s_{k}}}\right) \leq-2 \pi p_{0} d_{k} I\left(\frac{1}{\varepsilon_{n}}\left(\log \frac{1}{\varepsilon_{n}}\right)^{-\frac{1}{s_{k}}}\right)+2 \pi p_{0} d_{k}^{2} I\left(\left(\log \frac{1}{\varepsilon_{n}}\right)^{\frac{1}{s_{k}}}\right)+ \\
2 \pi p_{0} d_{k} I\left(\frac{1}{\varepsilon_{n}^{\alpha}}\left(\log \frac{1}{\varepsilon_{n}}\right)^{-\frac{1}{s_{k}}}\right)+2 \pi\left(p_{0} d_{k}+\frac{\alpha_{k}}{4}\left(\log \frac{1}{\varepsilon_{n}}\right)^{-1}\right) I\left(\frac{1}{\varepsilon_{n}^{1-\alpha}}\right)+O(1) .
\end{gathered}
$$

By assumption (H2) and (15) in Lemma 4, we deduce that the functional $I$ is increasing, thus for $n$ large enough, we get

$$
\begin{gathered}
I\left(\frac{1}{\varepsilon_{n}}\left(\log \frac{1}{\varepsilon_{n}}\right)^{-\frac{1}{s_{k}}}\right) \geq I\left(\frac{1}{\varepsilon_{n}^{\alpha}}\left(\log \frac{1}{\varepsilon_{n}}\right)^{-\frac{1}{s_{k}}}\right), \\
I\left(\frac{1}{\varepsilon_{n}}\left(\log \frac{1}{\varepsilon_{n}}\right)^{-\frac{1}{s_{k}}}\right) \geq I\left(\left(\log \frac{1}{\varepsilon_{n}}\right)^{\frac{1}{s_{k}}}\right)
\end{gathered}
$$

and

$$
I\left(\frac{1}{\varepsilon_{n}}\left(\log \frac{1}{\varepsilon_{n}}\right)^{-\frac{1}{s_{k}}}\right) \geq I\left(\frac{1}{\varepsilon_{n}^{1-\alpha}}\right) .
$$

Hence, by (87), the leading term of the second member in (95) is the negative one and we can conclude that

$$
h\left(R_{n}\right)-h\left(\left(\log \frac{1}{\varepsilon_{n}}\right)^{-\frac{1}{s_{k}}}\right) \rightarrow-\infty \text { as } n \rightarrow+\infty .
$$

This is a contradiction with (94) and arguing as in [3], (94) directly implies (74).

Step 2. Let $\eta$ as in (72) and $T_{\mathcal{E}_{n}}$ as in (45). We know that $B_{\eta}\left(b_{k}\right)$ contains exactly $d_{k}$ bad discs $B_{\lambda \varepsilon}\left(y_{j}\right)$, $j \in I_{k}$ satisfying (74).

We have

$$
\begin{aligned}
E_{\varepsilon_{n}}\left(u_{\varepsilon_{n}}, B_{\eta}\left(b_{k}\right)\right) & \geq E_{\varepsilon_{n}}\left(u_{\varepsilon_{n}}, B_{\eta}\left(b_{k}\right) \backslash B_{T_{\varepsilon_{n}}}\left(b_{k}\right)\right)+\sum_{j \in I_{k}} E_{\varepsilon_{n}}\left(u_{\varepsilon_{n}}, B_{T_{\varepsilon_{n}}}\left(b_{k}\right) \backslash B_{\lambda \varepsilon_{n}}\left(y_{j}\right)\right) \\
& =E_{1}+E_{2} .
\end{aligned}
$$

By Proposition 1, we have

$$
E_{1} \geq 2 \pi p_{0} d_{k}^{2} \log \frac{\eta}{T_{\varepsilon_{n}}}-2 \pi p_{0} d_{k}^{2} I\left(\frac{\eta}{T_{\varepsilon_{n}}}\right)-d_{k} C_{6} .
$$


where $C_{6}$ is a constant depending only on $C_{0}$.

Then

$$
E_{1} \geq 2 \pi p_{0} \frac{d_{k}^{2}}{s_{k}} \log \log \frac{1}{\varepsilon_{n}}-2 \pi p_{0} d_{k}^{2} I\left(\left(\log \frac{1}{\varepsilon_{n}}\right)^{\frac{1}{s_{k}}}\right)+O(1)
$$

By (40) in Remark 1 applied to $y_{1}, \ldots, y_{d_{k}}$, as $\left.v_{j}=\operatorname{deg}\left(u_{\varepsilon}, \partial B\left(y_{j}, \lambda \varepsilon\right)\right)\right)=+1$ for every $j=1, \ldots, d_{k}$ and by (68), we have

$$
E_{2} \geq 2 \pi p_{0} d_{k}\left(\log \frac{T_{\varepsilon_{n}}}{\lambda \varepsilon_{n}}-I\left(\frac{T_{\varepsilon_{n}}}{\lambda \varepsilon_{n}}\right)\right)+\frac{9}{8} \pi p_{0} \sum_{i \neq j} \log \frac{T_{\varepsilon_{n}}}{\left|y_{i}-y_{j}\right|}-C_{7}
$$

where $C_{7}$ is a constant depending only on $d_{k}, C_{0}$, and $p_{0}$ where $C_{0}$ is introduce in (66). Then

$$
\begin{aligned}
E_{2} \geq & -2 \pi p_{0} \frac{d_{k}}{s_{k}} \log \log \frac{1}{\varepsilon_{n}}+2 \pi p_{0} d_{k} \log \frac{1}{\varepsilon_{n}}-2 \pi p_{0} d_{k} I\left(\frac{1}{\varepsilon_{n}}\left(\log \frac{1}{\varepsilon_{n}}\right)^{-\frac{1}{s_{k}}}\right) \\
& +\frac{9}{8} \pi p_{0} \sum_{i \neq j} \log \frac{T_{\varepsilon_{n}}}{\left|y_{i}-y_{j}\right|}+O(1) .
\end{aligned}
$$

By collecting together (98) and (99) we obtain

$$
\begin{aligned}
E_{\varepsilon_{n}}\left(u_{\varepsilon_{n}}, B_{\eta}\left(b_{k}\right)\right) \geq & 2 \pi p_{0} \frac{d_{k}^{2}-d_{k}}{s_{k}} \log \log \frac{1}{\varepsilon_{n}}-2 \pi p_{0} d_{k}^{2} I\left(\left(\log \frac{1}{\varepsilon_{n}}\right)^{\frac{1}{s_{k}}}\right)+2 \pi p_{0} d_{k} \log \frac{1}{\varepsilon_{n}} \\
& -2 \pi p_{0} d_{k} I\left(\frac{1}{\varepsilon_{n}}\left(\log \frac{1}{\varepsilon_{n}}\right)^{-\frac{1}{s_{k}}}\right)+\frac{9}{8} \pi p_{0} \sum_{i \neq j} \log \frac{T_{\varepsilon_{n}}}{\left|y_{i}-y_{j}\right|}+O(1) .
\end{aligned}
$$

Summing over $k$ we have

$$
\begin{aligned}
E_{\varepsilon_{n}}\left(u_{\varepsilon_{n}}\right) \geq & E_{\varepsilon_{n}}\left(u_{\varepsilon_{n}}, \bigcup_{k=1}^{N} B_{\eta}\left(b_{k}\right)\right) \geq 2 \pi p_{0} d \log \frac{1}{\varepsilon_{n}}+2 \pi p_{0} \sum_{k=1}^{N} \frac{d_{k}^{2}-d_{k}}{s_{k}} \log \log \frac{1}{\varepsilon_{n}} \\
& -2 \pi p_{0} \sum_{k=1}^{N} d_{k}^{2} I\left(\left(\log \frac{1}{\varepsilon_{n}}\right)^{\frac{1}{s_{k}}}\right)-2 \pi p_{0} d I\left(\frac{1}{\varepsilon_{n}}\left(\log \frac{1}{\varepsilon_{n}}\right)^{-\frac{1}{s_{k}}}\right) \\
& +\frac{9}{8} \pi p_{0} \sum_{k=1}^{N} \sum_{i \neq j} \log \frac{T_{\varepsilon_{n}}}{\left|y_{i}-y_{j}\right|}+O(1)
\end{aligned}
$$

which is (73).

Remark 2. In Proposition 5 we have proved (73) for a star shaped domain. An argument of del Pino and Felmer in [12] can now be used to show that (66) holds without the assumption on the starshapedness of G. Hence (73) is still true for general domain and we can conclude again by acting as in [15].

\subsection{Proof of Theorem 1 Completed}

By collecting together Propositions 4 and 5, and taking into account Remark 2, we obtain Proposition 3 which is (10) of Theorem 1.

Thanks to estimate (66), we can now follow the construction of bad discs as in [5] and prove convergence (9) of Theorem 1. Since the arguments are identical to those of [5] we omit the details. Now Theorem 1 is completely proved.

Finally as a consequence of (64) and (100), we get the following estimate of the distance between the centers of bad discs. 
Corollary 2. For every $i \neq j$ in $I_{k}\left(1 \leq k \leq N_{2}\right)$ with $\left|I_{k}\right|=d_{k}>1$, we have

$$
\exp \left(-C_{8} I\left(\left(\log \frac{1}{\varepsilon_{n}}\right)^{\frac{1}{s_{k}}}\right)\right)\left|\log \varepsilon_{n}\right|^{-\frac{1}{s_{k}}} \leq\left|y_{i}-y_{j}\right| \leq C_{9}\left|\log \varepsilon_{n}\right|^{-\frac{1}{s_{k}}}
$$

where $C_{8}$ and $C_{9}$ are two constants independent of $\varepsilon$.

Proof. By lower bound (100) we have

$$
\begin{aligned}
\int_{\Omega} p|\nabla u|^{2} d x \geq & 2 \pi p_{0} d_{k} \log \frac{1}{\varepsilon_{n}}+2 \pi p_{0} \frac{d_{k}^{2}-d_{k}}{s_{k}} \log \log \frac{1}{\varepsilon_{n}}-2 \pi p_{0} \Sigma_{1}^{N} d_{k}^{2} I\left(\left(\log \frac{1}{\varepsilon_{n}}\right)^{\frac{1}{s_{k}}}\right) \\
& -2 \pi p_{0} d I\left(\frac{1}{\varepsilon_{n}}\left(\log \frac{1}{\varepsilon_{n}}\right)^{-\frac{1}{s_{k}}}\right)+\frac{9}{8} \pi p_{0} \sum_{i \neq j} \log \frac{T_{\varepsilon_{n}}}{\left|y_{i}-y_{j}\right|}+O(1) .
\end{aligned}
$$

The upper bound (64) and (103), imply

$$
\sum_{i \neq j} \log \left(\frac{\left|\log \varepsilon_{n}\right|^{-\frac{1}{s_{k}}}}{\left|y_{i}-y_{j}\right|}\right) \leq C_{8} I\left(\left(\log \frac{1}{\varepsilon_{n}}\right)^{\frac{1}{s_{k}}}\right)
$$

which by using (74), is the claimed result.

Author Contributions: Conceptualization, R.H. and C.P.; methodology, R.H. and C.P validation, R.H. and C.P.; formal analysis, R.H. and C.P. ; investigation, R.H. and C.P.; writing, original draft preparation, R.H. and C.P.; writing, review and editing, R.H. and C.P.; supervision, R.H. and C.P. All authors have read and agree to the published version of the manuscript.

Funding: This research received no external funding.

Conflicts of Interest: The authors declare no conflict of interest.

\section{References}

1. Alama, S.; Bronsard, L. Vortices and pinning effects for the Ginzburg-Landau model in multiply connected domains. Commun. Pure Appl. Math. 2006, 59, 36-70. [CrossRef]

2. Alama, S.; Bronsard, L.; Giorgi, T. Vortex structures for an $\mathrm{SO}(5)$ model of high-TC superconductivity and antiferromagnetism. Proc. R. Soc. Edinb. Sect. A Math. 2000, 130, 1183-1215, . [CrossRef]

3. Andre, N; Shafrir, I. Asymptotic behaviour of minimizers for the Ginzburg-Landau functional with weight, Parts I and II. Arch. Ration. Mech. Anal. 1998, 142, 45-73, 75-98. [CrossRef]

4. Bauman, P.; Peng, G. Analysis of minimizers of the Lawrence-Doniach energy for superconductors in applied fields. Discrete Contin. Dyn. Syst. Ser. B 2019, 24, 5903-5926. [CrossRef]

5. Bethuel, F.; Brezis, H. ; Hélein, F. Asymptotic for the minimization of a Ginzburg-Landau functional. Calc. Var. Partial. Differ. Equations 1993, 1 123-148. [CrossRef]

6. Bethuel, F.; Brezis, H.; Hélein, F. Ginzburg-Landau Vortices, Birkhäuser; Springer: Berlin, Germany, 1994.

7. Beaulieu, A. Hadiji, R. Asymptotic for minimizers of a class of Ginzburg-Landau equation with weight. C. R. Acad. Sci. Paris Sér. I Math. 1995, 320, 181-186, .

8. Beaulieu, A.; Hadiji, R. A Ginzburg-Landau problem having minima on the boundary. Proc. R. Soc. Edinb. Sect. A Math. 1998, 128, 123-148. [CrossRef]

9. Beaulieu, A.; Hadiji, R. Asymptotic behaviour of minimizers of a Ginzburg-Landau equation with weight near their zeros. Asymptot. Anal. 2000, 22, 303-347, .

10. Brezis, H.; Merle, F.; Rivière, T. Quantization effects for $-\Delta u=u\left(1-|u|^{2}\right)$ in $\mathbf{R}^{2}$. Arch. Ration. Mech. Anal. 1994, 126, 35-58,. [CrossRef]

11. DeGennes, P.G. Superconductivity of Metals and Alloys; Perseus Books: New York, NY, USA; Amsterdam, The Netherlands, 1996. 
12. Del Pino, M.; Felmer, P. Local minimizers for the Ginzburg-Landau energy. Math. Z. 1997, 225, 671-684. [CrossRef]

13. Du, Q.; Gunzburger, M. A model for supraconducting thin films having variable thickness. Physica D 1994, 69, 215-231. [CrossRef]

14. Hadiji, R.; Perugia, C. Minimization of a quasi-linear Ginzburg-Landau type energy. Nonlinear Anal. 2009, 71, 860-875. [CrossRef]

15. Hadiji, R.; Shafrir, I. Minimization of a Ginzburg-Landau type energy with potential having a zero of infinite order. Differ. Integral Equations 2006, 10, 1157-1176, and Erratum. Differ. Integral Equ. 2018, 31, 157-159.

16. Hadiji, R.; Shafrir, I. Minimization of a Ginzburg-Landau type energy with a particular potential. In Nonlinear Phenomena with Energy Dissipation; Gakuto Internat. Serv. Math. Sci. Appl.; Gakkotosho: Tokyo, Japan, 2008, Volume 29, pp. 141-151.

17. Han, Z.G.; Shafrir, I. Lower bounds for the energy of $S^{1}$-valued maps on perfored domains. J. Anal. Math. 1995, 66, 295-305. [CrossRef]

18. Jerrard, R. Lower bounds for generalized Ginzburg-Landau functionals. SIAM J. Math. Anal. 1999, 30, 721-746. [CrossRef]

19. Kurzke, M. Compactness results for Ginzburg-Landau type functionals with general potentials. Electron. J. Differ. Equ. 2010, 28, 35.

20. Rubinstein, J. On the equilibrium position of Ginzburg-Landau vortices. Zeitschrift für angewandte Mathematik und Physik ZAMP 1995, 46, 739-751. [CrossRef]

21. Sandier, E. Lower bounds for the energy of unit vector fields and applications. J. Funct. Anal. 1998, 152, 10.1006/jfan.1997.3170. [CrossRef]

22. Serfaty, S.; Tice, I. Ginzburg-Landau vortex dynamics with pinning and strong applied currents. Arch. Ration. Mech. Anal. 2011, 201, 413-464. [CrossRef]

23. Struwe, M. On the asymptotic behaviour of minimizers of the Ginzburg-Landau model in 2 dimensions. Differ. Int. Equations 1994, 7, 1613-1624, Erratum. Differ. Int. Equ. 1995, 8, 124.

(C) 2020 by the authors. Licensee MDPI, Basel, Switzerland. This article is an open access article distributed under the terms and conditions of the Creative Commons Attribution (CC BY) license (http:/ / creativecommons.org/licenses/by/4.0/). 